\title{
The innovative technologists and formation of the technical operation system of civil aircraft avionics
}

\author{
Sergey Kuznetsov ${ }^{1 *}$ \\ ${ }^{1}$ Moscow State Technical University of Civil Aviation, Moscow, Pulkovskaya 6a, Russia
}

\begin{abstract}
The system of technical operation (TO) of on-board equipment or avionics of aircraft (AC) as an object of research is a set of objects and means of technical operation, maintenance and repair programs, as well as personnel performing procedures and organizing the processes of TO. The quality of the TO system is manifested in the TO process - a set of processes of intended use, operational control, maintenance, restoration and repair. The TO process, as a process of changing the TO states, can be considered as a controlled random process, determined on the set of operation states by probabilistic characteristics. The quality of the TO system is characterized by a set of properties that determine its ability to satisfy with the maximum economic efficiency the needs of the TO system of the aircraft, while ensuring the required levels of reliability and readiness of the avionics for operation. That is, the TO system has all the features inherent in complex technical systems - a hierarchical ramified structure, subordination of goals and restrictions, wide interrelationships in the process of functioning. The main research apparatus in solving problems of optimization of processes and synthesis of TO systems is the apparatus of the theory of mathematical modeling of complex technical systems, based on a system approach, the integration of analytical and simulation modeling. The article solves the problem of determining the values of the parameters of the TO system such that the costs of the system during the TO process reach a minimum when all the required tasks are completed and all restrictions on the system's own parameters and indicators of its technical efficiency are met.
\end{abstract}

\section{Introduction}

Technical operation of the system (STE) of the aircraft - a scoop of facilities and maintenance funds, maintenance programs and repair, as well as the personnel performing the procedures and processes of organizing the technical operation of the Armed Forces [12].

Onboard equipment as technical operation object is a scoop of measuring, computing, controlling systems and display systems intended for manual tasks solutions, automated,

* Corresponding author: $\underline{\text { s.kuznetsov @ mstuca.aero }}$ 
semi-automatic and automatic piloting from takeoff to landing and delivery of information to consumers [3-6].

The equipment can be considered at several levels of detail, determined by the objectives of the research. Equipment combined into systems as a scoop and functional systems. Outside the board equipment is demountable blocks, apparatus and devices which, in turn, may be considered as the scoop of constructive-functional modules. The complexes combined in solution of a variety of flight and navigation problems on the basis of multifunctional system and wide interconnections [7-12].

Functional systems (FS), as integral parts of the complexes, are combined by solving specific flight and navigation problems on the basis of structural-block integration and wide inter-block connections.

Constructive and functional blocks (or line-replaceable units LRU), as components of the FS, are combined by solving particular problems of a certain function of the system within the framework of a construct that provides autonomous installation of the unit on board and removal from the board.

Constructive-functional modules (CFM), as components of the LRU, are designed to solve particular problems of a certain function of the block within the framework of the construct, which ensures the installation of the CFM into the block and its dismantling from the block.

Finally, electrical radioelements (ERE), as components of the CFM, are elementary structurally complete units that ensure the functioning of the CFM and do not provide for non-destructive assembly and dismantling works.

The means of technical operation of on-board equipment are means of aerodrome maintenance, restoration, control, tools and devices, as well as consumables designed to solve the problems of technical operation of objects installed on board the aircraft (complexes , FS, LRU) and objects dismantled from board (LRU, KFM, ERE).

Aerodrome facilities provide power and hydropower on the ground and are part of general aircraft facilities. Control facilities include airborne, ground-based and groundbased facilities, as well as control and test equipment. The means of recovery on board the aircraft are LRU . Recovery means unmounting 's on board LRU are KFM and ERE.

Maintenance and repair programs (MRP) define methods and strategies of TO, volumes, frequency and control technology, restoration, maintenance and repair, enshrined in the relevant specifications and technical documentation. Program MRP on -board equipment are part of the program MRP of the aircraft. Flight crew (FC) and engineering staff (ES) carry out procedures and organize maintenance processes in accordance with the requirements of operating manuals and normative technical documentation in order to ensure flight safety and high cost efficiency.

Quality of TO system manifested in the process of TO - scoop processes intended use, operational control, maintenance, repair and overhaul.

The use of onboard equipment for its intended purpose in flight includes work in preparatory unloaded modes, ensuring readiness for immediate use, and work in the main loaded modes, ensuring the performance of functional tasks. The use of onboard equipment for its intended purpose leads to the occurrence of failures and malfunctions, which are revealed in the processes of operational control: in flight, post-flight, pre-flight control and control of dismantled equipment. Operational control is an integral part of maintenance, which is divided into operational and periodic, seasonal, special and storage maintenance. Another part of maintenance in an operating enterprise is recovery - disaster and preventive. Avionics repair (overhaul, regulated, according to technical condition) is carried out at repair plants and manufacturing plants.

The technical operation of the aircraft and its on-board equipment can be considered as a process with a sequential change in the operating states. As such states, when used for the 
intended purpose, the states of operability, serviceability, malfunction and inoperability can be distinguished. In-flight monitoring states are in-flight monitoring, post-flight and preflight monitoring and dismantled equipment monitoring for serviceable, serviceable, inoperative or faulty equipment. Similarly, the states of operational and periodic maintenance, emergency and preventive recovery, and the state of repair can be distinguished. When real-time process research is required, consideration should be given to waiting, idle, and storage states.

The TO process as a process of changing the TO states proceeds in time under the influence of many factors that are random and non-random in nature. These include the occurrence of failures and malfunctions, the quality of MRP, the reliability of the controls, the availability of spare parts. Transitions of the TO process from state to state occur both at random and at deterministic times. Such transitions are generated by streams of events (for example, streams of failures, recoveries, control procedures, etc.). That is, the TO process can be considered as a random process determined on the set of operation states by the probabilistic characteristics of the transitions. This allows solving the problems of analyzing the process and the TO system using a well-developed apparatus of the theory of random processes.

The TO process is inherently a controlled process. The possibility of intervention in this process is both objective deterministic, due to the operation manual and the maintenance program, and subjective random nature, due to illegal or erroneous actions of flight crew and engineering staff.

That is, the TO process can be considered as a controlled random process determined by a set of control actions and external disturbances with probabilistic characteristics. This allows solving the problems of optimization of the process and synthesis of the system using the apparatus of the theory of controlled random processes and the theory of automatic control.

\section{Formulation of the problem}

Hierarchy of the TO system of avionics. In relation to avionics TO system, the system of a higher hierarchy level is the TO system of aircraft. Quality of aircraft TO system is characterized by a scoop of properties that determine its capacity as cost-effectively meet the needs of the air transport system (ATS), while ensuring the required levels of reliability and availability of the aircrafts to the operation.

The avionics TO system is subordinate to the aircraft TO system, which defines its purpose and limitations. Quality of the avionics TO system characterized by scoop of properties that determine its ability to comply with the maximum economic efficiency needs the aircraft TO system, while providing required at humectant reliability and availability of avionics for operation. In relation to avionics TO system, systems of a lower level of the hierarchy are systems of maintenance, repair, and operational control. The avionics TO system defines the goals and limitations of these systems.

Thus, the avionics TO system has all the features inherent in complex technical systems, namely: a hierarchical ramified structure, subordination of goals and restrictions, wide interrelationships in the process of functioning. This allows us to conclude that the formation and improvement of the avionics TO system should be carried out on the basis of a comprehensive system analysis of the processes occurring in it using modern mathematical methods of the theory of complex systems.

Hierarchy of efficiency criteria for the avionics TO system and related systems. In accordance with the hierarchy of the objectives of the functioning of the aircraft TO system and the avionics TO system, we construct a hierarchy of criteria for the effectiveness of these 
systems. To do this, it is necessary to formalize the tasks they solve, characterize their parameters, as well as the processes occurring in them.

Let $\Omega_{\text {to }}^{\text {ac }}$ is the set of tasks solved by the aircraft TO system. The system of a higher level of the hierarchy - the aviation transport system (ATS), presents many required tasks for the implementation of the aircraft TO system $\left(\Omega_{\text {to }}^{\mathrm{ac}}\right)^{*}$.

Let $\Gamma_{\text {to }}^{\mathrm{ac}}=\left\{\gamma_{\text {to }}^{\mathrm{ac}}(\mathrm{j})\right\}$ is the set of parameters of the aircraft TO system, determined by its structure, connections, resources and characteristics. The set of constraints on the parameters of the aircraft TO system $\left(\Gamma_{\text {to }}^{\mathrm{ac}}\right)^{*}=\left[\left\{\gamma_{\mathrm{to}}^{\mathrm{ac}}(\mathrm{j})\right\}^{*}\right]$ sets the boundaries for changing the parameters $\Gamma_{\text {to }}^{\mathrm{ac}}(\mathrm{j})$ based on the conservatism of structures and connections, limited resources and the maximum attainability of the characteristics of the aircraft TO system.

The process of TO of the aircraft $\xi_{\text {to }}^{\mathrm{ac}}\left(\mathrm{t}, \Gamma_{\mathrm{to}}^{\mathrm{ac}}\right)$ as a function of time $t$ and a set of parameters $\Gamma_{\text {to }}^{\text {ac }}$ determines the efficiency of the aircraft TO system, which is manifested in economic and technical indicators. Let $\mathrm{C}_{\text {to }}^{\mathrm{ac}}$ is an indicator of the economic efficiency of the aircraft TO system, determined in the process of TO, for example, operating costs.

The set of values of indicators of technical efficiency of the aircraft TO system will be denoted $\mathrm{R}_{\text {to }}^{\mathrm{ac}}=\left\{\mathrm{r}_{\mathrm{to}}^{\mathrm{ac}}(1)\right\}$. Each indicator $\mathrm{r}_{\mathrm{to}}^{\mathrm{ac}}(1)=\mathrm{r}_{\mathrm{to}}^{\mathrm{ac}}\left(\mathrm{t}, \Gamma_{\text {to }}^{\mathrm{ac}}\right)$ is a function of time and many parameters $\Gamma_{\text {to }}^{\mathrm{ac}}$. The system of a higher level of the ATS hierarchy presents $\Gamma_{\text {to }}^{\mathrm{ac}}$ with the required values of a set of indicators of technical efficiency $\left(\mathrm{R}_{\mathrm{to}}^{\mathrm{ac}}\right)^{*}=\left[\left\{\mathrm{r}_{\mathrm{to}}^{\mathrm{ac}}(1)\right\}^{*}\right]$.

Then the criterion for the effectiveness of the aircraft TO system is the objective function and constraints

$$
\begin{aligned}
& \min _{\gamma_{\mathrm{to}}^{\mathrm{ac}}(\mathrm{j}) \in \Gamma_{\text {to }}^{\mathrm{ac}}(\mathrm{j})}\left[\mathrm{C}_{\mathrm{to}}^{\mathrm{ac}}\left\{\xi_{\text {to }}^{\mathrm{ac}}\left(\mathrm{t}, \Gamma_{\text {to }}^{\mathrm{ac}}\right)\right\}\right], \\
& \left(\Omega_{\text {to }}^{\mathrm{ac}}\right)^{*}-\Omega_{\text {to }}^{\mathrm{ac}}=\varnothing,\left(\Omega_{\text {to }}^{\mathrm{ac}}\right)^{*} \in\left(\Omega_{\mathrm{ats}}\right)^{*} \\
& \gamma_{\text {to }}^{\mathrm{ac}}(\mathrm{j}) \geq \leq\left\{\gamma_{\text {to }}^{\mathrm{ac}}(\mathrm{j})\right\}^{*}, \quad \gamma_{\text {to }}^{\mathrm{ac}}(\mathrm{j}) \in \Gamma_{\text {to }}^{\mathrm{ac}} \text {, } \\
& \mathrm{r}_{\text {to }}^{\mathrm{ac}}(\mathrm{j}) \geq \leq\left\{\mathrm{r}_{\text {to }}^{\mathrm{ac}}(\mathrm{j})\right\}^{*}, \quad \mathrm{r}_{\text {to }}^{\mathrm{ac}}(\mathrm{j}) \in \mathrm{R}_{\text {to }}^{\mathrm{ac}}, \quad\left\{\mathrm{R}_{\text {to }}^{\mathrm{ac}}\right\}^{*} \in \mathrm{R}_{\text {ats }}^{*} \text {. }
\end{aligned}
$$

That is, the criterion for the effectiveness of the aircraft TO system is the achievement of the minimum value of the indicator of economic efficiency on a given set of system parameters in the process of TO of the aircraft, provided that all the tasks required by the ATS are fulfilled and all restrictions on the parameters of the system and indicators of its technical efficiency are met.

The avionics TO system solves many tasks $\Omega_{\text {to }}^{\mathrm{a}}$. The aircraft TO system as a system of a higher level of the hierarchy presents many tasks to be performed $\left(\Omega_{\text {to }}^{\mathrm{a}}\right)^{*}$, moreover $\left(\Omega_{\text {to }}^{\mathrm{a}}\right)^{*} \in\left(\Omega_{\mathrm{to}}^{\mathrm{a}}\right)^{*}$.

The avionics TO system has many parameters $\Gamma_{\text {to }}^{\mathrm{a}}=\left\{\gamma_{\text {to }}^{\mathrm{a}}(\mathrm{j})\right\}$ determined by its structure, connections, resources and characteristics. Since structures and connections are conservative, resources are limited, and characteristics cannot exceed certain limit values, the set $\Gamma_{\text {to }}^{\mathrm{a}}$ is within the boundaries of the set $\left(\Gamma_{\text {to }}^{\mathrm{a}}\right)^{*}=\left[\left\{\gamma_{\text {to }}^{\mathrm{a}}(\mathrm{j})\right\}^{*}\right]$ for all $\mathrm{j}$. 
The process of TO of avionics $\xi_{\text {to }}^{\mathrm{a}}\left(\mathrm{t}, \Gamma_{\text {to }}^{\mathrm{a}}\right)$ embedded in the To process of the aircraft $\xi_{\text {to }}^{\mathrm{a}}$ and is a function of time $\mathrm{t}$ and the set of parameters $\Gamma_{\text {to }}^{\mathrm{a}}$.

The economic efficiency of the avionics TO system is characterized by operating costs $C_{\text {to }}^{\mathrm{a}}$ determined in the process of $\mathrm{TO} \xi_{\text {to }}^{\mathrm{a}}\left(\mathrm{t}, \Gamma_{\text {to }}^{\mathrm{a}}\right)$. The technical efficiency of the avionics TO system is characterized by a variety of indicators $\mathrm{R}_{\mathrm{to}}^{\mathrm{a}}=\left\{\mathrm{r}_{\mathrm{to}}^{\mathrm{a}}(1)\right\}$, and each indicator $\mathrm{r}_{\mathrm{to}}^{\mathrm{a}}(\mathrm{l})=\mathrm{r}_{\mathrm{to}}^{\mathrm{a}}\left(\mathrm{t}, \Gamma_{\text {to }}^{\mathrm{a}}\right)$ is a function of time $t$ and a set of parameters $\Gamma_{\text {to }}^{\mathrm{a}}$. The aircraft TO system, as a system of a higher level of hierarchy, presents the avionics TO system with the required values of a set of indicators of technical efficiency $\left(\mathrm{R}_{\mathrm{to}}^{\mathrm{a}}\right)^{*}=\left[\left\{\mathrm{r}_{\mathrm{to}}^{\mathrm{a}}(1)\right\}\right]^{*}$.

The criterion for the efficiency of the avionics TO system is the minimum operating costs for a given set of system parameters in the $\mathrm{TO}$ of avionics when all the tasks required by the aircraft TO system are fulfilled and all restrictions on the parameters of the system and indicators of its technical efficiency are met.

$$
\begin{aligned}
& \min _{\gamma_{\text {стэ }}^{\mathrm{a}}(\mathrm{j}) \in \Gamma_{\text {стэ }}^{\mathrm{a}}(\mathrm{j})}\left[\mathrm{C}_{\text {to }}^{\mathrm{a}}\left\{\xi_{\text {to }}^{\mathrm{a}}\left(\mathrm{t}, \Gamma_{\text {to }}^{\mathrm{a}}\right)\right\}\right], \\
& \left(\Omega_{\mathrm{to}}^{\mathrm{a}}\right)^{*}-\Omega_{\mathrm{to}}^{\mathrm{a}}=\varnothing,\left(\Omega_{\mathrm{to}}^{\mathrm{a}}\right)^{*} \in\left(\Omega_{\mathrm{to}}^{\mathrm{ac}}\right)^{*}, \\
& \gamma_{\text {Tо }}^{\mathrm{a}}(\mathrm{j}) \geq \leq\left\{\gamma_{\text {TO }}^{\mathrm{a}}(\mathrm{j})\right\}^{*}, \quad \gamma_{\text {Tо }}^{\mathrm{a}}(\mathrm{j}) \in \Gamma_{\text {то }}^{\mathrm{a}}, \\
& \mathrm{r}_{\text {to }}^{\mathrm{a}}(\mathrm{j}) \geq \leq\left\{\mathrm{r}_{\text {to }}^{\mathrm{a}}(\mathrm{j})\right\}^{*}, \quad \mathrm{r}_{\text {to }}^{\mathrm{a}}(\mathrm{j}) \in \mathrm{R}_{\text {to }}^{\mathrm{a}}, \quad\left\{\mathrm{R}_{\text {to }}^{\mathrm{a}}\right\}^{*} \in\left\{\mathrm{R}_{\text {to }}^{\mathrm{a}}\right\}^{*} .
\end{aligned}
$$

Thus, the hierarchy of efficiency criteria for the aircraft TO system and the avionics TO system is fulfilled.

Statement of the general problem and its decomposition into composite problems. Based on the analysis of the avionics TO system as an object of research, the analysis of mathematical modeling as a research apparatus and the developed hierarchy of criteria for the effectiveness of the avionics TO system and the systems interacting with it, we will formulate the general problem as follows.

On a given set of parameters of the avionics TO system, determine the values of the parameters such that the costs of the system during the TO process reach a minimum when all the required tasks are completed and all restrictions on the system's own parameters and indicators of its technical efficiency are met.

To solve a common problem with the help of innovative technologies, it is necessary to consistently solve the following problems:

- analyze the processes of TO of the operated and newly commissioned avionics on the basis of operational statistics using modern statistical methods for the subsequent substantiation of mathematical models;

- develop mathematical models of the characteristics of the TO processes, for which to modify the traditional methods for determining the parameter of the flow of failures and the function of failure rate;

- develop mathematical models of the processes and avionics TO system based on the results of the analysis of operational statistics and to carry out the appropriate refinement of the mathematical apparatus, taking into account the features of the models;

- develop mathematical models to optimize the processes of TO with the deepening of the capabilities of the mathematical apparatus and to carry out optimization;

- develop mathematical models for the synthesis of avionics TO systems with the deepening of the capabilities of the mathematical apparatus and to carry out the synthesis.

Obtained in the study of theoretical results can serve as a scientific basis for solving practical problems of formation and perfection of the avionics TO systems using innovative technologies. 


\section{The solution of the problem}

Analytical and simulation modeling of processes and avionics TO systems. Formation and improvement of systems for the technical operation of avionics is a long and laborious process that requires the use of the most advanced mathematical methods. This is due to the fact that, firstly, avionics TO systems are complex systems, for the analysis of which general mathematical and engineering methods have not yet been created. Secondly, the quality of the avionics TO systems manifests itself over a fairly long time interval, often covering years. The required quality is achieved only at the stationary stage of the technical operation process. The control actions on the system are very limited due to its conservatism. In addition, the system itself has a high cost, and any changes in it can affect flight safety. Thus, mathematical modeling of the avionics TO system should significantly reduce the costs of forming the system, since it allows at the early stages of its operation to introduce certain adjustments based on available and constantly updated information.

The best results can usually be achieved with the integrated application of analytical and simulation modeling, followed by pilot operation to assess the effectiveness of the avionics TO systems. The complex method built on this basis is called theoretical-experimental or computational-experimental.

The essence of this method is that the performance indicators of the system and the main characteristics of the quality of subsystems are determined theoretically on a special complex of analytical and simulation models, and obtaining a number of initial data necessary for modeling, checking the degree of proximity of the models to the real system, as well as checking the functioning of the systems are carried out in the process of its trial operation.

This method makes it possible to evaluate both the quality characteristics of individual subsystems and the performance indicators of the system as a whole using a single set of models interconnected in terms of input and output characteristics. The computational and experimental method for assessing the efficiency of the avionics TO systems includes:

1) analysis of the system, TO methods and maintenance and repair strategies, quantitative and qualitative characteristics of the system;

2) selection and justification of the main parameters, characteristics and quality indicators of the system and its subsystems, determination of their interdependence;

3 ) analysis of the possibility of obtaining the necessary initial information and available computational - modeling base;

4) determination of the rational sequence, stage and general scheme for assessing the characteristics of the system;

5) building a general topological model of the system, carrying out its decomposition, distributing tasks between models and justifying the required composition of models;

6) substantiation of the requirements for the input and output characteristics of the models and for the accuracy of their determination, the choice of the structure, the procedure for creating and the method of implementing the models of the complex;

7) selection and substantiation of methods for approximating models to real simulated subsystems and determination of the experimental information necessary for this;

8) selection of conditions for conducting and planning tests in the process of experimental and serial operation, justification of the required volume of experimental and serial operational statistics;

9) development of software and mathematical support, development and implementation of analytical and simulation models on computing tools;

10) organizing the collection of experimental and serial operational statistics with an assessment of the degree of proximity of models to real systems and processes, correction of models; 
11) carrying out mathematical modeling on corrected models, determining the quality characteristics of software and mathematical support;

12) determination of the quality characteristics of the TE BO system with an assessment of their accuracy and reliability, development of recommendations for their improvement.

In the process of creating models, the following alternative often arises: either create one complex model to evaluate all the necessary and sometimes heterogeneous characteristics on it at once, or create several simpler models - each to determine one or several homogeneous characteristics. Experience shows that preference should be given to the second solution, since this way is more realistic and gives serious savings in time, labor and computational resources.

A systematic approach to the problem of synthesis of avionics TO systems. A systematic approach to determining the structure of a complex of models for assessing the effectiveness of the avionics TO systems is to comply with the following basic provisions:

1) knowledge of the behavior of the subsystems that form the system is still insufficient to determine the behavior of the system as a whole, until the details of the connections are clarified;

2) any given behavior of the system can be carried out by an infinitely large number of possible connections of its parts, that is, the behavior of the system does not unambiguously determine the connections between subsystems;

3 ) in a real system, the nature of the connections depends on how many inputs and outputs of subsystems are used.

The application of these provisions in assessing the effectiveness of the avionics TO systems allows us to draw several practical conclusions.

First, to assess the quality indicators of the system as a whole, it is not enough to assess the quality indicators of subsystems. An apparatus is also needed that allows, when recalculating these estimates of indicators into an estimate of the quality index of the system, it is enough to accurately reproduce the details of the connections and relationships between subsystems.

Secondly, no matter how accurately the interconnections between the subsystems are reproduced in assessing the system quality indicator, there is no guarantee that with a different combination of external conditions the same effect of the system's behavior will not be obtained with a different nature of the subsystem connections. Therefore, the system modeling apparatus must be capable of obtaining the necessary estimates in the entire range of operating conditions of its subsystems.

Thirdly, when creating models of a system and its subsystems, it is necessary to focus on the minimum number of indicators and characteristics that should be obtained on a given model, since this makes it possible to construct a model most simply taking into account the minimum number of connections between subsystems.

Fourthly, in order to obtain identical outputs on several models of the same system and on the system itself, it is necessary that all inputs and outputs of the corresponding connections of the subsystem models and the real system strictly coincide.

The general scheme of mathematical modeling should provide the following order:

1) some of the mathematical models must be created and tested even before the operation of the system begins;

2 ) the models should be refined as the system is developed and tested;

3 ) by the beginning of the final stage of modeling, the models must be isomorphic to the real modeled system and real processes;

4) all created models should be a single complex, that is, each model should take a certain place among other models and be in certain relationships with them, the nature of which depends on the tasks to be solved. 
Considering these provisions, the following main categories of requirements can be presented to each of the created models:

- by the tasks being solved;

- by the number and type of output characteristics or indicators;

- on the accuracy and reliability of the assessment of these characteristics and indicators;

- under the conditions in which the model should allow modeling and obtaining reliable estimates.

As a result of the analysis of the requirements for the model, first of all, the question of the choice of this or that type of model, the method of development and the way of implementing its elements is resolved.

Requirements for mathematical models of avionics TO systems. The main requirements for mathematical models are the following:

1) ensuring the assessment of the studied characteristics in a wide range of operating conditions of the system;

2) the required quality of formalization of the assessment by comparing the simulation results with operational statistics;

3) practically acceptable performance of the model program and ensuring the required amount of modeling in the shortest possible time;

4) the presence of a developed system of service programs for displaying, registering, processing and analyzing the simulation results;

5) the relative invariance of algorithms and programs to changes in the original data.

Mathematical models of avionics TO systems should solve the following tasks:

1) evaluation of the main decisions contained in the programs and plans of maintenance and repair;

2) assessment of the influence of the characteristics of the fuel cell system on the indicators of its efficiency;

3 ) determination of possible ranges of changes in the operating conditions of the system (homogeneous and heterogeneous fleet of aircraft, type of operating enterprise, etc.);

4) justification of the requirements for the accuracy of the assessment of characteristics;

5) identification of additional capabilities of the system for solving new problems.

Modeling principles. The principles of constructing algorithms and programs of the mathematical model of the system should not only ensure that the main factors affecting the efficiency of the system are taken into account, but also, to a certain extent, "materialize" the following basic methodological principles for assessing efficiency:

1) the assessment of the efficiency and the system is carried out using a mathematical model with the required characteristics in terms of reliability and speed and the necessary "service" when displaying, registering and processing simulation results;

2) the initial data on the characteristics of the main processes are obtained from the results of autonomous modeling on private mathematical models;

3 ) the conditions and scope of autonomous modeling must ensure the required accuracy of evaluating performance indicators on the mathematical model of the system;

4) the structure and parameters of the models are refined as information about the system as a whole is accumulated based on the results of trial operation.

Such a scheme for assessing the effectiveness of the system ensures the transfer of the main burden of work on the reliable formalization of the entire system to private models, which provide the bulk of autonomous modeling in a wide range of possible conditions for the functioning of the system. Autonomous modeling results are used to obtain input data for a mathematical model of the entire system. In addition, an automatic assessment of the sensitivity of the model output indicators is provided both to the initial data for modeling and to the parameters of the model blocks, which characterize the accuracy of formalization of the corresponding processes. This makes it possible to evaluate the errors in calculating 
performance indicators due to the inaccuracy of the initial data obtained on particular models, as well as to solve the problem of ensuring the balance of accuracy as a ratio between formalization errors and errors in the initial data when constructing a system model.

Stages of modeling. As the experience of the development and practical use of mathematical models for assessing the effectiveness of complex systems shows, the life cycle of these models includes a number of objectively existing stages, determined both by the level of knowledge about the system and by the specification of modeling tasks at various stages of the formation of the system. The main steps are as follows.

At the first stage, the development of the first edition of the algorithms for the input and functionally necessary blocks of the mathematical model is carried out based on the initial data on the characteristics of the system. At the same stage, autonomous programs of functionally necessary blocks, programs of special mathematical software for modeling (special standard programs) are developed and their preliminary docking is carried out through the general control program of the model and arrays of basic variables (arrays of exchange information). As a result of the work of the first stage, a cooperation of executors of algorithms and programs is formed and a software-algorithmic reserve is created, which ensures further development of a mathematical model with the required characteristics of quality and speed.

At the second stage, the algorithms of the functionally necessary blocks of the model (second edition) are modified according to the supplemented initial data about the system. At this stage, the output group of model blocks is developed. Algorithms and programs are being refined based on the results of autonomous modeling of private models, and models are being developed in full staffing.

At the third stage, the algorithms and programs of the model are refined in the process of carrying out preliminary simulations. The maximum possible performance characteristics of the model are achieved. Preliminary estimates of the efficiency of the system are obtained. As a result of the work of the second and third stages, by the end of the preliminary tests of the model, its development is completed in full staffing and the required characteristics of its performance are obtained, ensuring the required amount of modeling in a given time.

At the fourth stage, the model is finalized according to the results of preliminary tests. The modeling of the system is carried out in the required volume with obtaining estimates of its effectiveness in a wide range of conditions of its use, with an assessment of accuracy and reliability.

Thus, the main requirements for the mathematical models of the processes and systems of TE BO are formulated. The tasks, principles and stages of modeling are determined.

\section{Conclusions}

1. The avionics TO system as the object of study is the scoop of facilities and maintenance funds, maintenance programs and repair, as well as the personnel performing the procedures and organizes the process of avionics TO.

2. The quality of the avionics TO system is shown in the TO processes intended use, operational control, maintenance, repair and overhaul. A TO process as a process of changing TO states proceeds in time under the influence of many factors that are random and deterministic. That is, a TO process can be considered as a controlled random process determined on the set of operation states by the probabilistic characteristics of transitions and on the set of control actions and external disturbances with probabilistic characteristics.

3. The quality of avionics TO system is characterized by a scoop of properties that determine its ability to comply with the maximum economic efficiency of the aircraft TO system needs, while ensuring the required levels of reliability and availability of avionics to be in use. That is, the avionics TO system has all the features inherent in complex 
technical systems - a hierarchical ramified structure, subordination of goals and restrictions, wide interrelationships in the process of functioning.

4. The features of digital avionics as an object of operation, the features of the means of TO of the digital avionics and the peculiarities of the organization of the processes of the TO of the new generation of the aircrafts and digital avionics significantly affect the processes of forming the avionics TO system and should be appropriately taken into account when constructing mathematical models.

5. The main research apparatus in solving the problems of optimization of processes and synthesis of avionics TO system is the apparatus of the theory of mathematical modeling of complex technical systems, based on a systematic approach, the integration of analytical and simulation modeling, which is actually innovative.

6. The task is to determine the values of the parameters of the avionics TO system such that the costs of the system in the process of TO would reach a minimum while performing all the required tasks and observing all restrictions on the system's own parameters and indicators of its technical efficiency.

\section{References}

1. S.V. Kuznetsov, The Science Bulletin MSTUCA Volume 20 №1 132-140 (2017)

2. S.V. Kuznetsov, The Science MSTUCA Bulletin Vol 20 №6 15-24 (2017)

3. E.A. Fedosov, V.V. Kosyanchuk, N.I. Sel'vesyuk Radioelectronic Technology № 1 66-71 (2015)

4. V.S. Kulabuhov, Aerospace Instrument №12 11-31 (2015)

5. E.A. Fedosov, Prospective directions for the development of onboard equipment for civil aircraft 6-14 (2017)

6. V.B. Poliakov, E.S. Neretin, A.S. Ivanov, A.S. Budkov, S.A. Djachenko, S.O. Dudkin, Proceedings MAI №100 (2018)

7. Sreerupa Das, IEEE Aerospace Conference (2015)

8. Alexandru Prisacaru, Przemyslaw Jakub Gromala, Mateus Bagetti Jeronimo, Bongtae Han and Guo Qi Zhang Thermal Mechanical and Multi-Physics Simulation and Experiments in Microelectronics and Microsystems (EuroSimE) 18th International Conference 1-11 (2017)

9. Nastaran Soltanipour, Sadegh Rahrovani, John Martinsson and Robin Westlund, IEEE 23rd International Conference on Intelligent Transportation Systems (ITSC) (2020)

10. Jian Cao, Xin Fu, Xigao Fang, Yi Hu, Guirong Zhou and Huamin Jia IEEE CSAA Guidance, Navigation and Control Conference (CGNCC) (2018)

11. Panagiotis Korvesis, Stephane Besseau and Michalis Vazirgiannis IEEE 34th International Conference on Data Engineering (ICDE) (2018)

12. Andreas Zeitler IEEE / AIAA $38^{\text {th }}$ Digital Avionics Systems Conference (DASC) (2019) 